\title{
New Mathematical Model of an Inverter-Based Generator for Stability Studies of Microgrid Systems
}

\author{
F. Andrade, L. Romeral \\ Motion Control and Industrial Applications Group \\ Departament d'Enginyeria Electrònica \\ Universitat Politècnica de Catalunya \\ 08222 Terrassa. Catalonia. Spain \\ Fabio.andrade@mcia.upc.edu
}

\begin{abstract}
A new mathematical model of a renewable generator, with a DC-AC interface, based on the concept of electrostatic machine is presented. This new model has a direct relationship between the DC and AC side. Moreover, it can be used for stability studies, taking into account the dynamics of the DC link and to find saturations and limits on the control signals.
\end{abstract}

Keywords - Microgrid control, Microgrid model, stability of Microgrids

\section{NOMENCLATURE}

$v_{f d}, v_{f q}:$

Instantaneous electrostatic field voltage in dq0-axis.

$v_{d}, v_{q}, v_{0}:$

$i_{f d,} i_{f q}:$

$i_{d}, i_{q}, i_{0}$ :

Instantaneous electrostatic stator voltage in dq0-axis.

Electrostatic field current in dq-axis.

Instantaneous electrostatic stator currents in dq0-axis.

$C_{f f d}, C_{f f d}: \quad$ Self-capacitance of rotor in dq-axis.

$C_{d}, C_{q}, C_{o}$ :

$C_{f d-d}, C_{f q-q}$ :

Self-capacitance of stator in dq0-axis

"Mutual" capacitances between stator and rotor surfaces.

$R: \quad$ Armature resistances.

$R_{f}: \quad$ Armature resistances.

$Q_{d}, Q_{q}, Q_{0}$ : $\quad$ Demand charge in each dq0-circuit.

$Q_{f d}, Q_{f q}: \quad$ Available charge of rotor in dq0-axis.

$\Theta$ :

Angle by d-axis leads the axis of a-phase.

$\omega_{r}:$

\section{INTRODUCTION}

Distributed generation systems have been improved to increase the efficiency, the power-sharing controls and other feature from different control strategies. These control strategies are tuned and analyzed from a set of equations which describes the generator's behavior. The grouping of generators and consumers under the Microgrid concept has allowed the small-generators to be better utilized.

Renewable generating systems with DC-AC converters give a greater degree of freedom in the control. Their control units could trade off the delivered power using global strategies and re-configure the strategy if it is required. Conventional droop control [1-3] has been quite used to share the consumer's power between different generators without any

\author{
K. Kampouropoulos, J. Cusido \\ Area of Energy \\ Fundació CTM Centre Tecnològic \\ 08242 Manresa. Catalonia. Spain \\ konstantinos.kampouropoulos@ctm.com.es
}

communication. Furthermore, different modifications have been proposed towards improving the dynamic characteristics. In [4], a control system that uses an inner control loop in gridconnected mode and three different control strategies to operate in island mode is proposed (conventional droop, power quality mode and sync mode). Although inverter-based generators do not have an important inertia, P/F-droop control has been used widely to control the power [5-11]. On the other hand, P/Vdroop controls that are presented in $[12,13]$ uses a line impedance that is considered mainly as resistive. Moreover, [12] presents a combination of the $\mathrm{P} / \mathrm{V}$-droop with an outer $\mathrm{Vg} / \mathrm{Vdc}$-droop control to improve the stability area.

This paper presents a mathematical model of a renewable generator with DC-AC interface, based on the concept of electrostatic machine. This new model has a direct relationship between the $\mathrm{DC}$ and $\mathrm{AC}$ side.

The outline of the paper is presented as follows. The next section describes the mathematical model of electrostatic machine. In section IV, the relationships between the electrostatic machine and the power converter interface are presented; in section $\mathrm{V}$ a Microgrid system with a perturbation in the DC-link is presented. Finally, Section VI concludes by highlighting the main contributions of the paper.

\section{CONCEPT OF ELECTROSTATIC MACHINE}

The concept of electrostatic machine is proposed by introducing the transformation of the DC-side and the threephase inverter of Figure 1 in a rotating reference frame (DQ0). Then, the inverter and the DC-link can be considered as generators of a rotating electric field inside of a virtual electrostatic machine. The stator circuits consist of two-axis armature capacitors carrying an electric charge Q.

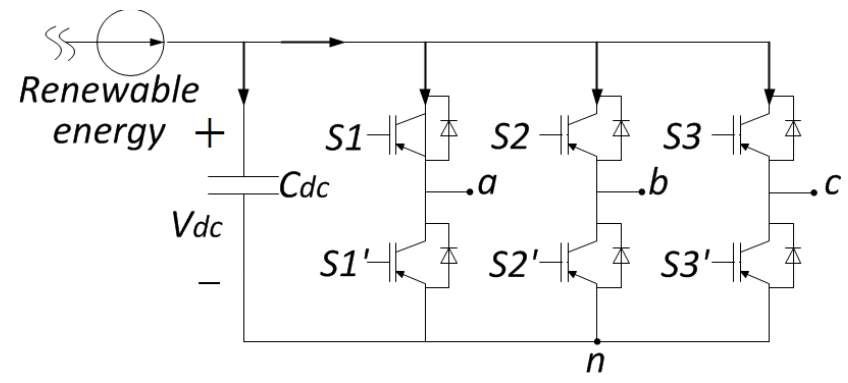

Figure 1. Power electronic interfaces 


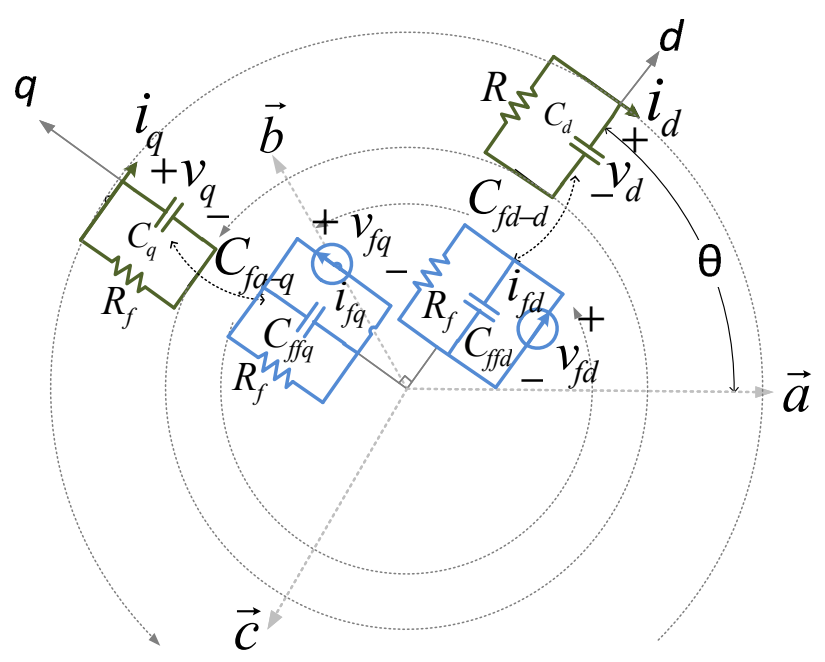

Figure 2. equivalent electrostatic machine

\section{A. Mathematical Description}

It is considered that the electrostatic machine is physically equivalent to a magnetic synchronous machine with a pair of field poles. The rotor is supplied by a direct voltage, which produces an electric field that induces charges in the armature circuit. The electric field magnitude and the speed of rotation are function of the DC capacitor, the modulation index and the frequency of the PWM. Figure 2 depicts the equivalent system.

The current equations of the stator circuits in the electrostatic machine can be described as:

$i_{d}=\frac{d}{d t} Q_{d}-Q_{q} \frac{d \theta}{d t}-\frac{v_{d}}{R}$

$i_{q}=\frac{d}{d t} Q_{q}+Q_{d} \frac{d \theta}{d t}-\frac{v_{q}}{R}$

$i_{0}=\frac{d}{d t} Q_{0}-\frac{v_{0}}{R}$

The charge in each circuit at any instant is given by:

$$
\begin{aligned}
& Q_{d}=-C_{d} v_{d}+C_{f d-d} v_{f d} \\
& Q_{q}=-C_{q} v_{q}+C_{f q-q} v_{f q} \\
& Q_{0}=-C_{0} v_{0}
\end{aligned}
$$

Where $C_{f d-d}$ and $C_{f q-q}$ are the equivalent capacitance between the rotor and the stator in dq-axes and the $\left(C_{\left.a f d-d * v_{f d}\right)}\right.$ product is equivalent to the transfer charge from the rotor to the stator.

The current equations of the rotor in the electrostatic machine are:

$$
\begin{aligned}
& i_{f d}=\frac{d}{d t} Q_{f d}-\frac{v_{f d}}{R_{f}} \\
& i_{f q}=\frac{d}{d t} Q_{f q}-\frac{v_{f q}}{R_{f}}
\end{aligned}
$$

The equivalent charges $Q_{f d}$ and $Q_{f q}$ are given by:

$$
\begin{aligned}
& Q_{f d}=C_{f f d} v_{f d}-\frac{3}{2} C_{f d-d} v_{d} \\
& Q_{f q}=C_{f f q} v_{f q}-\frac{3}{2} C_{f q-q} v_{q}
\end{aligned}
$$

\section{B. Per Unit Representation}

In order to normalize the system's variables, a per unit transformation has been used. In this transformation, the line to neutral voltage, the line's current and the grid frequency have been chosen as base quantities for the stator. The complete model is expressed as follows:

$$
\begin{aligned}
& i_{d}=\frac{d}{d t} Q_{d}-Q_{q} \omega-\frac{1}{R} v_{d} \\
& i_{q}=\frac{d}{d t} Q_{q}+Q_{d} \omega-\frac{1}{R} v_{q} \\
& i_{0}=\frac{d}{d t} Q_{0}-\frac{1}{R} v_{0}
\end{aligned}
$$

$i_{f d}=\frac{d}{d t} Q_{f d}+\frac{1}{R_{f}} v_{f d}$

$i_{f q}=\frac{d}{d t} Q_{f q}+\frac{1}{R_{f}} v_{f q}$

Where

$$
\begin{aligned}
& Q_{d}=-C_{d} v_{d}+C_{f d-d} v_{f d} \\
& Q_{q}=-C_{q} v_{q}+C_{f q-q} v_{f d} \\
& Q_{0}=-C_{0} v_{0} \\
& Q_{f d}=C_{f f d} v_{f d}-C_{f d-d} v_{d} \\
& Q_{f q}=C_{f f q} v_{f q}-C_{f q-q} v_{d}
\end{aligned}
$$

\section{EQUIVALENT OF DG BASED IN ELECTRONIC INTERFACED}

\section{A. General Description}

In Figure 3, a renewable source generator based on electronic power interface is depicted. This kind of generator has an outer control to work like a plug and play system. The generator is synchronized by means of a PLL block with the electric utility. Furthermore, droop curves are often used to share the active and reactive power in the Microgrid. The inner control is used to track the reference signals and the Space Vector Modulation (SVM) module to manage the states of the inverter. 


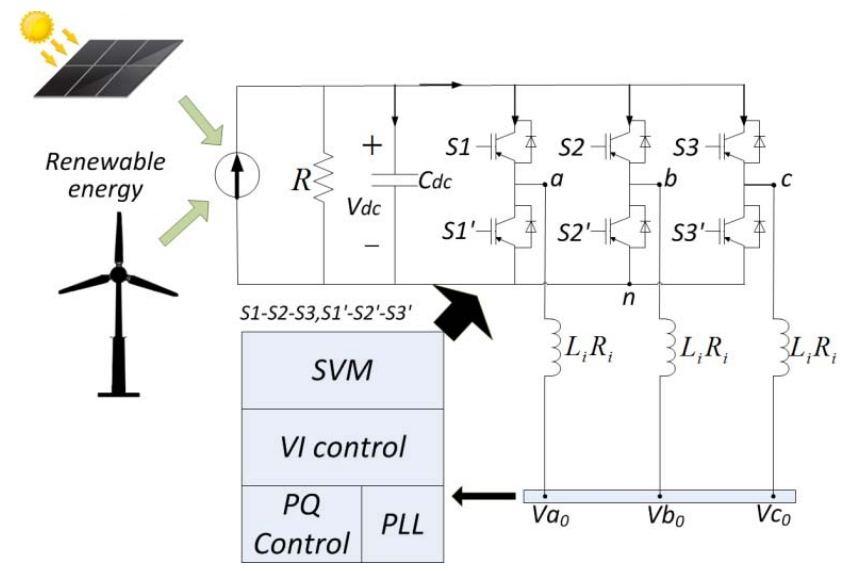

Figure 3. Power electronic interfaces

A renewable prime source, solar or wind, delivers energy to the DC link which is modeled by a current source. The DC voltage is regulated by means of the corresponding control block. DC-link and control systems could be seen like a rotating electric field which gives an electric charge to the three-phase system. The speed of the electric field is equal to the reference frequency in the inner loop of control that is changed according to the shared power by means of the droop curves.

Taking into account that the switching frequency in the SVM module is a decade higher than the working frequency, it is possible to use the fundamental harmonic of each signal to obtain continuous algebraic models and state equations of the plant.

\section{B. Equivalent Circuits for Direct and Quadrature Axes}

Equivalent circuits representing the complete characteristics, including the current equations, are shown in Fig. 4. These equivalent circuits do not take into account the output filter. The circuits represent electrical characteristics and provide a visual description of the power electronics interface.

\section{Steady-State Analysis}

The behavior of electronic interface under balanced steadystate conditions may be analyzed by applying the per unit equation (7) as it is summarized in the last section. Since the rotor's and the stator's quantities are constant under steady

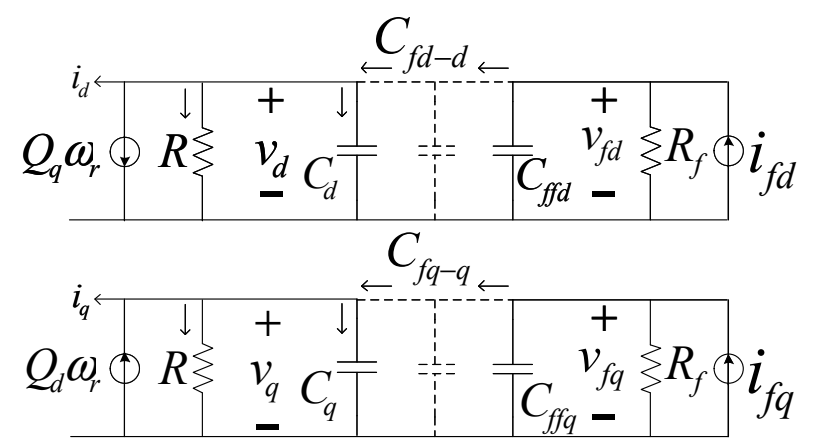

Figure 4 Equivalent circuits representing of the power electronic interface and renewable energy input state, all the time derivative terms drop out of the model. Also the zero-sequence components are not present and the $\omega_{r}$ it is considered as $1 \mathrm{pu}$.

Therefore, the per unit equations under balanced steadystate conditions can be described as:

$i_{d}=-Q_{q} \omega_{r}-\frac{1}{R} V_{d}$

$i_{q}=Q_{d} \omega_{r}-\frac{1}{R} V_{q}$

$i_{f d}=\frac{1}{R_{f}} V_{f d}$

$i_{f q}=\frac{1}{R_{f}} V_{f q}$

$Q_{d}=-C_{d} V_{d}+C_{f d-d} V_{f d}$

$Q_{q}=-C_{q} V_{q}+C_{f q-q} V_{f d}$

$Q_{f d}=C_{f f d} V_{f d}-C_{f d-d} V_{d}$

$Q_{f q}=C_{f f q} V_{f q}-C_{f q-q} V_{q}$

The above equations can be used to find the field voltages. Replacing the product $\left(-\omega_{r} C\right)^{-1}$ by the corresponding reactance $X c$, concludes that:

$$
\begin{aligned}
& V_{f d}=\left(\frac{1}{X c_{d}} V_{d}-\frac{1}{R} V_{q}-i_{q}\right) X c_{f d-d} \\
& V_{f q}=\left(\frac{1}{R} V_{d}+\frac{1}{X c_{d}} V_{q}+i_{q}\right) X c_{f q-q}
\end{aligned}
$$

Also, the stator's voltage and current may be written as phasor representation " $v_{t}$ " and " $i_{t}$ " where $\mathrm{v}_{t}=v_{d}+j v_{q}$ and $i_{t}=i_{d}+j i_{q}$. The relationships between each other according to (7) are:

$$
\begin{aligned}
& i_{d}=\frac{1}{X c_{f q-q}} V_{f q}-\frac{1}{X c_{q}} V_{q}+\frac{1}{R} V_{d} \\
& i_{q}=-\frac{1}{X c_{f d-d}} V_{f d}+\frac{1}{X c_{d}} V_{d}-\frac{1}{R} V_{q}
\end{aligned}
$$

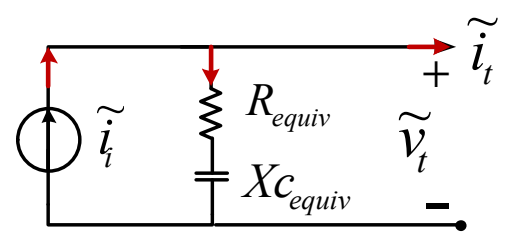

Figure 5 equivalent circuit

The equivalent circuit of the stator circuit is shown in Fig 5. The current's equations are:

$$
\begin{aligned}
& \tilde{i_{i}}=\tilde{i}_{t}+\left(\frac{1}{R_{\text {equiv }}}+j \frac{1}{X_{\text {equiv }}}\right) \cdot \tilde{v}_{t} \\
& \tilde{i_{t}}=\underbrace{\left(\frac{V_{f q}}{X c_{f q-q}}-\frac{V_{d}}{R}-\frac{V_{q}}{X c_{q}}\right)}_{i_{d}}+j \underbrace{(\underbrace{}_{f d} \frac{V_{f d}}{X c_{f d-d}}+\frac{V_{d}}{X c_{d}}+\frac{V_{q}}{R})}_{i_{q}} \\
& \tilde{v}_{t}=V_{d}+j V_{q}
\end{aligned}
$$




$$
\begin{aligned}
& \operatorname{Re}\left\{\widetilde{\tilde{i}_{t}}\right\}=\left(\frac{V_{f q}}{X c_{f q-q}}-\frac{V_{d}}{R}+\frac{V_{q}}{X c_{q}}\right)+\left(\frac{V_{d}}{R_{\text {equiv }}}-\frac{V_{q}}{X_{\text {equiv }}}\right) \\
& \operatorname{Re}\left\{\widetilde{i_{t}}\right\}=\frac{V_{f q}}{X c_{f q-q}}+\left(-\frac{V_{d}}{R}+\frac{V_{d}}{R_{\text {equiv }}}\right)+\left(\frac{V_{q}}{X c_{q}}-\frac{V_{q}}{X_{\text {equiv }}}\right)
\end{aligned}
$$

If $R=R_{\text {equiv }}$ and $X c_{q}=X c_{\text {equiv }}$ then

$\operatorname{Re}\left\{\tilde{i_{t}}\right\}=\frac{1}{X c_{f q-q}} V_{f q}$

The imaginary part:

$\operatorname{Im}\left\{\widetilde{i_{t}}\right\}=\left(-\frac{V_{f d}}{X c_{f d-d}}-\frac{V_{d}}{X c_{d}}-\frac{V_{q}}{R}\right)+\left(\frac{V_{d}}{X_{\text {equiv }}}+\frac{V_{q}}{R_{\text {equiv }}}\right)$

$\operatorname{Im}\left\{\widetilde{i}_{t}\right\}=-\frac{V_{f d}}{X c_{f d-d}}+\left(-\frac{V_{d}}{X c_{d}}+\frac{V_{d}}{X_{\text {equiv }}}\right)$

If $X c_{q}=X c_{d}$ then

$\operatorname{Im}\left\{\widetilde{\hat{i}_{t}}\right\}=-\frac{1}{X c_{f d-d}} V_{f d}$

Finally, the phasor is: $\widetilde{i_{t}}=\frac{1}{X c_{f q-q}} V_{f q}-j \frac{1}{X c_{f d-d}} V_{f d}$

So far, the power interfaces have been modeled assuming a DC source from the prime energy side. In this case, under steady-state, the DC bus is constant and its energy input is equal to the energy output. Additionally, the capacitor of the DC-bus has been considered as a rotating reactance along the d-axis. Thus,

$$
\begin{aligned}
& X c_{f d-d}=X c_{f q-q}=X c_{d c}=\left(\omega C_{d c}\right)^{-1} \\
& V_{f q}=\frac{V_{d c}}{2} d_{q} \quad V_{f d}=\frac{V_{d c}}{2} d_{d}
\end{aligned}
$$

Where $\mathrm{d}$ is the average duty cycle.

The equivalents resistance and admittance are

$$
\begin{aligned}
& X c_{\text {equiv }}=X c_{d c}\left(\frac{d_{q}^{2}-d_{d}^{2}}{d_{q}^{2}+d_{d}^{2}}\right) \\
& R_{\text {equiv }}=X c_{d c}\left(\frac{2 d_{q} d_{d}}{d_{q}^{2}+d_{d}^{2}}\right)
\end{aligned}
$$

\section{A MICROGRID SYSTEM}

A Microgrid system of two inverter-based generator operating in island mode is considered in fig. 6. Both generators have been working in steady state when a disturbance in the primary energy input (irradiance or wind) occurred to one of the generators. As soon as the disturbance appears, the DC link begins discharging and the controller tries to keep the reference signal. Each generator has a three phase H-bridge, a nominal DC voltage of $800 \mathrm{~V}$ and an output voltage of $230 \mathrm{~V} \mathrm{rms}$ of $50 \mathrm{~Hz}$ frequency. Simulations of mathematical and electrical models have been carried out by means of PSCAD X4 and Matlab/Simulink.

In steady-state, the Microgrid delivers power to a constant load. The generators share the power delivered through static f/P and V/Q droop-curves. Figure 7 shows the power delivered by each generator. The system begins with a transient and it is considered that the steady-state has been reached in $20 \mathrm{~s}$. The

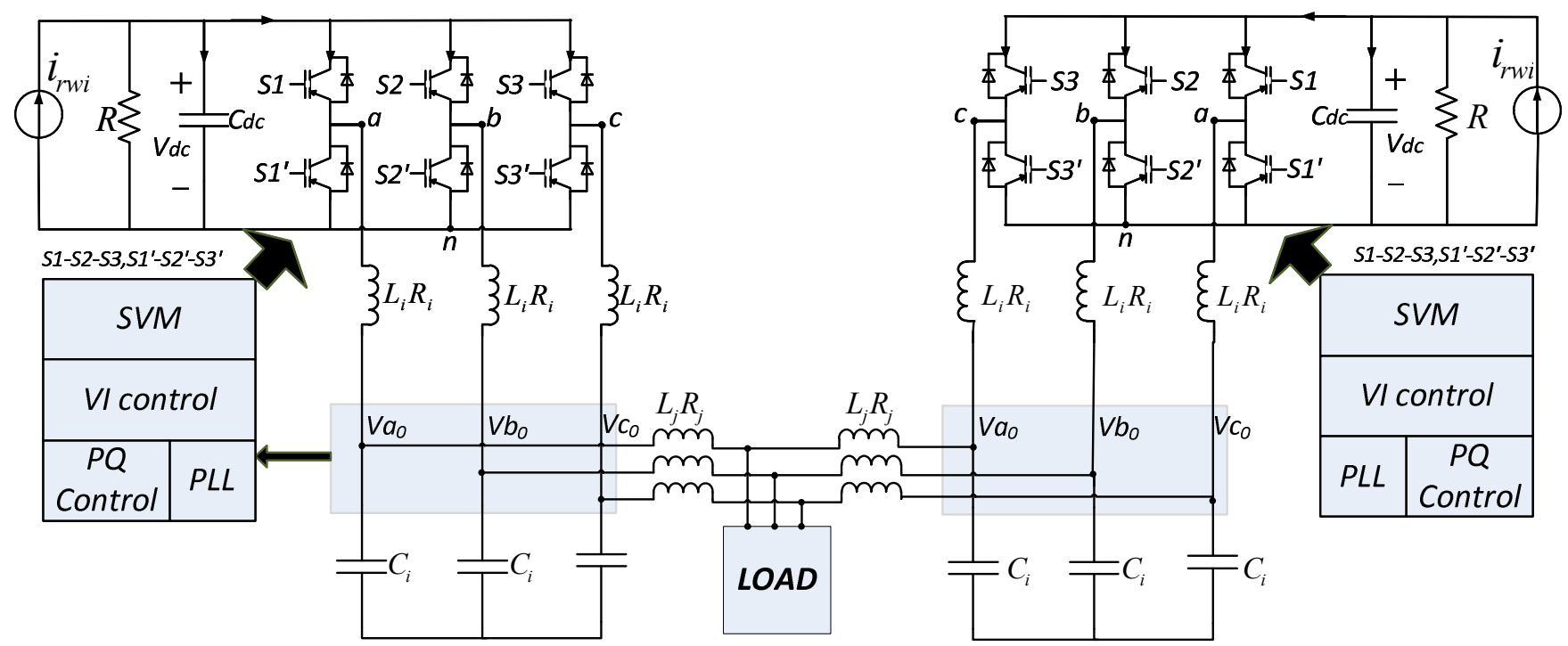

Figure 6 Microgrid system in alone mode 

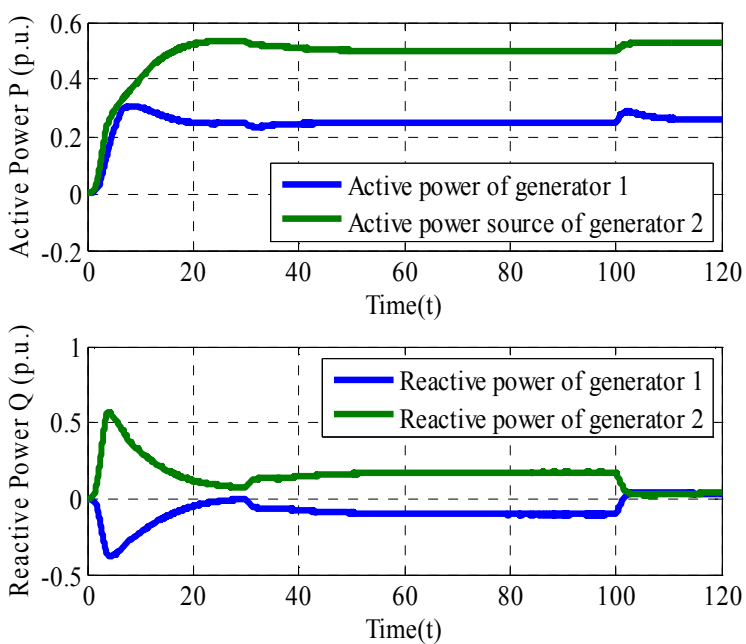

Figure 7 Microgrid sistem in alone mode

system has only delivered active power to the load. The disturbance of irradiance in the generator 1 occurs during the time of $30 \mathrm{~s}$. Figure 8 shows the DC-link of each generator. When the disturbance occurs, the dc-link of the generator 1 begins to decrease and this generator requires reactive power. The necessary reactive power is delivered by the generator 2 .

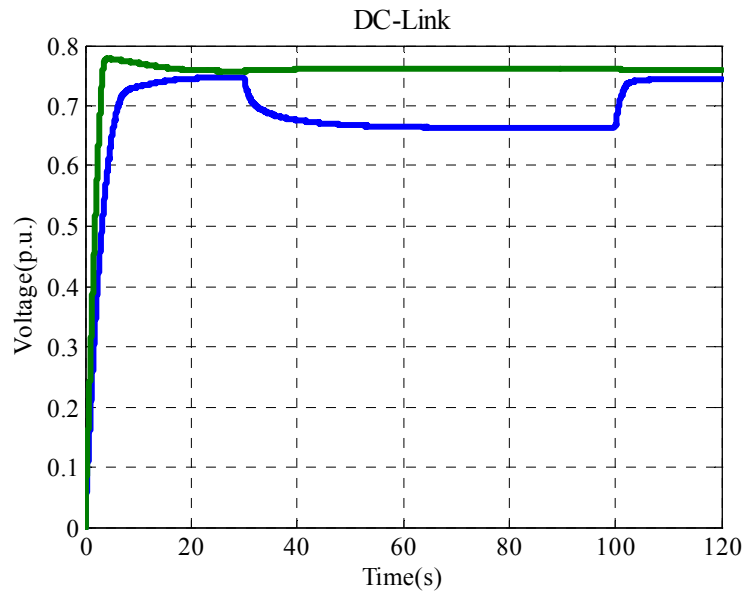

Figure 8. Microgrid sistem in alone mode
The DC-link of the generator 1 cannot be recovered because of the reached limit of saturation in the control's signal. However, because of the active power and the reactive power that is delivered and received (respectively) by the generator 1, the system is able to maintain the global stability.

Equivalent unfiled circuit of the Microgrid system according to the model exposed is illustrated in figure 9. The output filters, the line impedances, the load and the model of each generator are presented in the circuit. The values of resistors, capacitors and current sources of the model are functions of the DC-capacitance and control's signals $\left(d_{d}\right.$ and $\left.d_{q}\right)$.
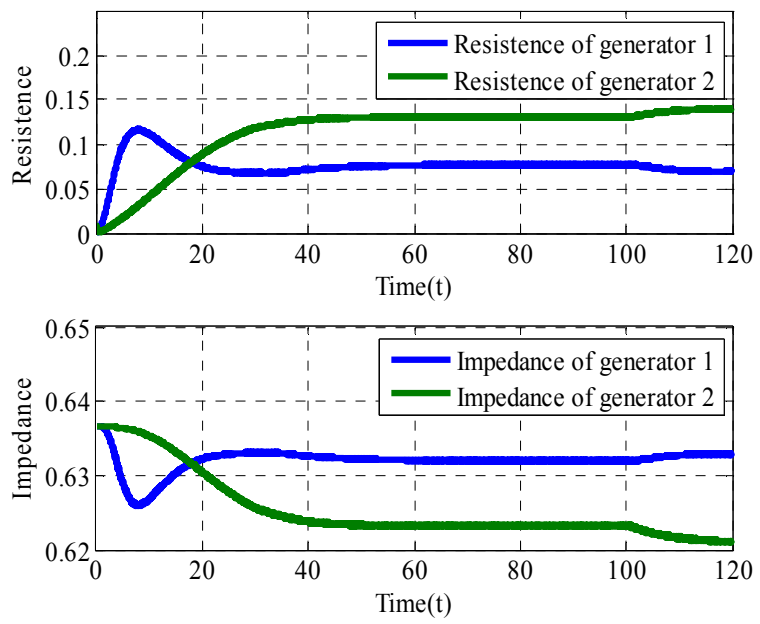

Figure 10. Microgrid sistem in alone mode

The behavior of the signals of the current's sources as well as the signals of resistance and impedance of each generator are shown in Figures 10 and 11.

In Figure 10, the Cartesian components of the current sources of each generator can be observed. The q-component of the generator 1 has a negative change in the time of $30 \mathrm{~s}$. This change indicates the saturation of the control signal. On the other hand, the resistance and impedance values vary depending on the active and reactive power delivered to the system (Figure 11).

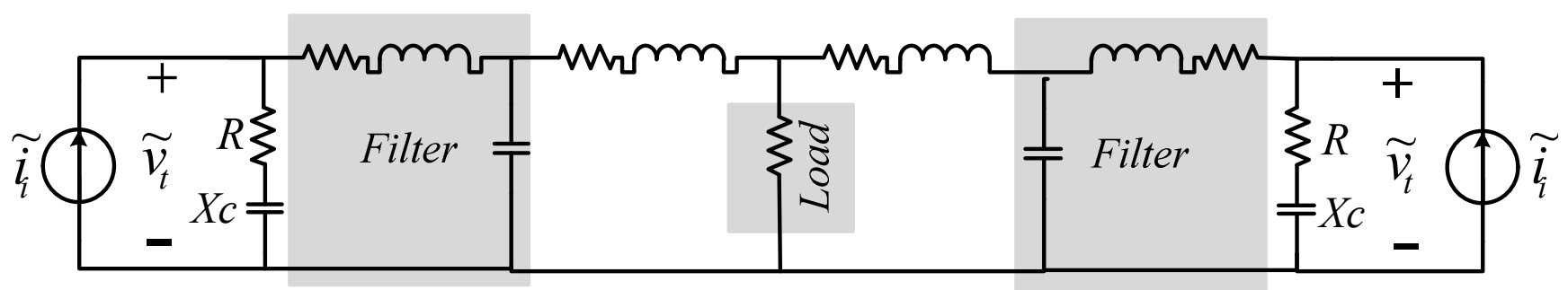

Figure 9. Microgrid sistem in alone mode 

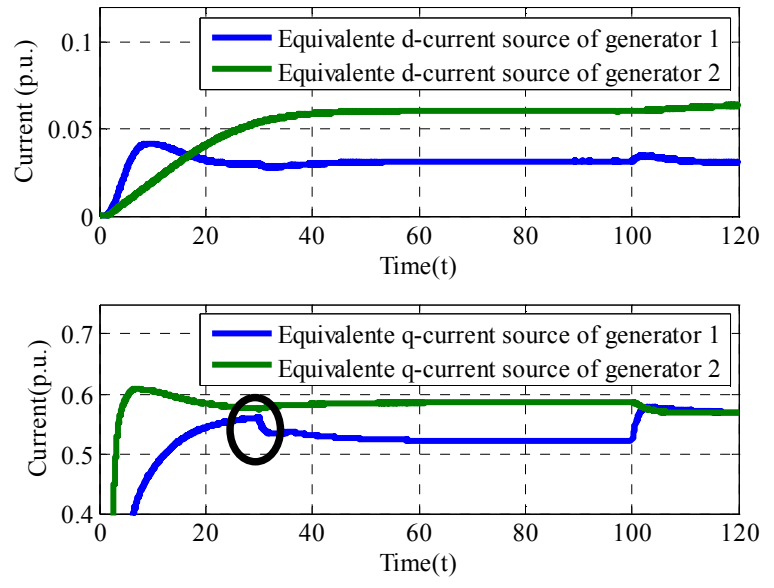

Figure 11. Microgrid sistem in alone mode

\section{CONCLUSIONES}

Many models of generators with power electronic interfaces have been used. These models use differential equations from output filter, the transfer functions of the controller to analyze their dynamics to small perturbations. Furthermore, the presented model allows analyzing the dynamics of DC-link, control signals and power flow behavior more directly. A mathematical model based on the concept of electrostatic machine is proposed.

\section{REFERENCES}

[1] M. N. Marwali, et al., "Control of distributed generation systems - Part II: Load sharing control," Ieee Transactions on Power Electronics, vol. 19, pp. 1551-1561, 2004.

[2] J. M. Guerrero, et al., "A wireless controller to enhance dynamic performance of parallel inverters in distributed generation systems," Ieee Transactions on Power Electronics, vol. 19, pp. 1205-1213, 2004.
[3] J. M. Guerrero, et al., "Wireless-control strategy for parallel operation of distributed-generation inverters," Ieee Transactions on Industrial Electronics, vol. 53, pp. 1461-1470, 2006.

[4] P. Arboleya, et al., "An improved control scheme based in droop characteristic for microgrid converters," Electric Power Systems Research, vol. 80, Oct 2010.

[5] T. L. Vandoorn, et al., "Active Load Control in Islanded Microgrids Based on the Grid Voltage," Smart Grid, IEEE Transactions on, vol. 2, pp. 139-151, 2011.

[6] Y. Mohamed and E. F. Ei-Saadany, "Adaptive Decentralized Droop Controller to Preserve Power Sharing Stability of Paralleled Inverters in Distributed Generation Microgrids," Ieee Transactions on Power Electronics, vol. 23, pp. 2806-2816, Nov 2008.

[7] E. Barklund, et al., "Energy Management in Autonomous Microgrid Using Stability-Constrained Droop Control of Inverters," Ieee Transactions on Power Electronics, vol. 23, pp. 2346-2352, Sep 2008.

[8] R. Majumder, et al., "Improvement of Stability and Load Sharing in an Autonomous Microgrid Using Supplementary Droop Control Loop," Ieee Transactions on Power Systems, vol. 25, May 2010.

[9] J. Kim, et al., "Mode Adaptive Droop Control With Virtual Output Impedances for an Inverter-Based Flexible AC Microgrid," Ieee Transactions on Power Electronics, vol. 26, Mar 2011.

[10] D. Georgakis, et al., "Operation of a prototype Microgrid system based on micro-sources equipped with fast-acting power electronics interfaces," Pesc 04: 2004 Ieee 35th Annual Power Electronics Specialists Conference, Vols 1-6, Conference Proceedings, pp. 25212526, 2004.

[11] M. Savaghebi, et al., "Secondary Control Scheme for Voltage Unbalance Compensation in an Islanded Droop-Controlled Microgrid," Smart Grid, IEEE Transactions on, vol. 3, pp. 797-807, 2012.

[12] T. L. Vandoorn, et al., "Analogy Between Conventional Grid Control and Islanded Microgrid Control Based on a Global DC-Link Voltage Droop," Ieee Transactions on Power Delivery, vol. 27, Jul 2012.

[13] J. M. Guerrero, et al., "Decentralized control for parallel operation of distributed generation inverters using resistive output impedance," Ieee Transactions on Industrial Electronics, vol. 54, pp. 994-1004, 2007. 